\title{
Iniciação sexual e práticas contraceptivas de adolescentes na tríplice fronteira entre Brasil, Argentina e Paraguai
}

\author{
Elis Maria T. Palma Priotto, ${ }^{1}$ Aline Luiza Führ, ${ }^{2}$ Ludmila Mourão Xavier Gomes ${ }^{2}$ e \\ Thiago Luis de Andrade Barbosa²
}

Como citar Priotto EMTP, Führ AL, Gomes LMX, Barbosa TLA. Iniciação sexual e práticas contraceptivas de adolescentes na tríplice fronteira entre Brasil, Argentina e Paraguai. Rev Panam Salud Pública. 2018;42:e16. https://doi.org/10.26633/RPSP.2018.16

RESUMO

Palavras-chave
Objetivo. Determinar a prevalência de iniciados sexualmente e as práticas contraceptivas de adolescentes na região de tríplice fronteira entre Foz do Iguaçu (Brasil), Ciudad del Este (Paraguai) e Puerto Iguazú (Argentina).

Método. Estudo transversal do qual participaram 2788 adolescentes de escolas públicas com idade entre 12 e 18 anos. As informações foram obtidas através de questionários aplicados nas escolas no período de 2012 a 2013. Foram analisadas a prevalência de iniciação sexual, práticas contraceptivas e variáveis sociodemográficas dos indivíduos dos três municípios. Para verificar a associação entre as variáveis, utilizou-se o qui-quadrado com nível de significância de 5\%.

Resultados. A prevalência geral de iniciação sexual dos adolescentes foi de 34,6\% (Puerto Iguazú: 45,3\%; Foz do Iguaçu: 35,2\%; Ciudad del Este: 23,3\%), sendo maior no sexo masculino e na faixa etária de 15 a 16 anos. Quanto a já ter tido iniciação sexual, esse fator associou-se a sexo, idade, escolaridade e turno de aula dos participantes. O recebimento de informações sobre contraceptivos foi associado a sexo, idade, escolaridade e uso de contraceptivo nos três municípios/países. Observou-se que os adolescentes de ambos os sexos haviam recebido informação sobre contraceptivos, principalmente da família e da escola.

Conclusões. Sugere-se que os municípios busquem a estruturação de política de fronteira trinacional com programas e projetos intersetoriais no intuito de promover práticas educativas voltadas à orientação da saúde sexual e reprodutiva de adolescentes.

Comportamento sexual; áreas de fronteira; comportamento contraceptivo; Argentina; Brasil; Paraguai.
Estudos $(1,2)$ mostram que os adolescentes em países de baixa e média renda apresentam maior vulnerabilidade a gravidez não planejada e concepção e a infecções sexualmente transmissíveis, como por HIV. Além disso, tanto em países desenvolvidos como naqueles em desenvolvimento, a maioria dos jovens

\footnotetext{
Universidade Estadual do Oeste do Paraná (UNIOESTE), Centro de Educação, Letras e Saúde (CELS), Campus Foz do Iguaçu (PR), Brasil. Correspondência: elispalmapriotto@hotmail.com
}

tem sua primeira relação sexual antes dos 18 anos (3); a Organização Mundial da Saúde (OMS) aponta os 15 anos como idade média de iniciação sexual dos adolescentes (4).

No Brasil, um estudo realizado no Rio de Janeiro observou que, entre adolescentes que tiveram relações sexuais antes

\footnotetext{
Universidade Federal da Integração LatinoAmericana (UNILA), Instituto Latino-Americano de Ciências da Saúde e da Natureza (ILACVN), Foz do Iguaçu (PR), Brasil.
}

dos 15 anos, 24,4\% eram portadores de infecções sexualmente transmissíveis e $7,8 \%$ apresentavam histórico dessas infecções (5). Nos Estados Unidos, um levantamento revelou que $41 \%$ dos adolescentes do ensino médio não haviam usado preservativo e $14 \%$ não haviam usado método contraceptivo durante a última relação sexual (6).

Um relatório de 2013 da Organização das Nações Unidas (ONU) apontou 
que, na região da América Latina, a iniciação sexual precoce está relacionada a elevadas taxas de fertilidade e ao acesso insuficiente à contracepção desde o início da vida sexual. Nesse mesmo relatório, o Brasil apresentou taxa de gravidez de $9,8 \%$ entre mulheres de 15 a 19 anos (7). O acesso insuficiente à contracepção tem provocado ainda aumento das infecções por HIV na América Latina, sendo que no Brasil foi observado incremento de $4 \%$ entre 2010 e 2015 (8).

Entretanto, permanecem lacunas na literatura sobre a sexualidade de adolescentes, em especial entre os que residem em regiões de fronteira. A região de tríplice fronteira entre Brasil, Argentina e Paraguai, por exemplo, é destaque no plano de relações internacionais por questões econômicas, culturais e geopolíticas. A mobilidade populacional na região influencia o cotidiano da fronteira e a construção de identidades socioculturais (9). Nesse contexto, a irregularidade de acesso a serviços de saúde, os conflitos culturais, a exposição a novos conceitos e preconceitos e a redes econômicas, sociais e sexuais têm influência na saúde do adolescente (8-10). Assim, o presente estudo objetivou avaliar a presença de iniciação sexual e as práticas contraceptivas em adolescentes na região da tríplice fronteira entre $\mathrm{Foz}$ do Iguaçu, Brasil, Ciudad del Este, Paraguai e Puerto Iguazú, Argentina.

\section{MATERIAIS E MÉTODOS}

Este estudo transversal foi realizado na região de tríplice fronteira entre os municípios de Foz do Iguaçu, no extremo oeste do Paraná (Brasil), Ciudad del Este (Paraguai) e Puerto Iguazú (Argentina). $\mathrm{O}$ estudo foi realizado no período de 2012 a 2013 em 75 escolas públicas: 26 em Foz do Iguaçu, 26 em Ciudad del Este e 23 em Puerto Iguazú. Os participantes da pesquisa foram adolescentes de ambos os sexos matriculados nessas instituições. Foram critérios de inclusão: ser estudante de escola pública, residir em um dos municípios do estudo e ter idade de 12 a 18 anos. A idade adotada no estudo teve como base o Estatuto da Criança e do Adolescente do Brasil, que abrange a fase da adolescência conforme a legislação de cada país (no Paraguai, maiores de 13 anos e menores de 18 anos; e na Argentina, entre 10 a 20 anos de idade) $(11,12)$.
No Brasil e na Argentina, todas as escolas públicas foram contatadas para participação na pesquisa. No Paraguai, as 127 escolas foram mapeadas e as escolas participantes foram selecionadas por sorteio. As escolas localizadas na área rural não foram incluídas no estudo.

O cálculo amostral baseou-se no método de amostragem estratificada e aleatorização dos indivíduos entrevistados a partir dos seguintes parâmetros: grau de confiança de $95 \%$ e erro amostral de 3\%. O tamanho da amostra foi calculado do total da população na faixa etária de 12 a 18 anos. Em Foz do Iguaçu, do total de 25123 alunos matriculados, foram selecionados 1018 adolescentes. Em Ciudad del Este, dos 8760 matriculados, 936 alunos foram selecionados. Finalmente, em Puerto Iguazú, do total de 3958 alunos matriculados, 856 adolescentes foram selecionados. Para minimizar eventuais perdas ou recusas, acrescentaram-se 30\% ao quantitativo calculado de adolescentes. A aleatorização dos indivíduos entrevistados se deu por meio de sorteio com base nas listas fornecidas pelas escolas. $\mathrm{O}$ número de participantes da pesquisa em cada escola foi calculado com base na partilha proporcional de alunos com idade de 12 a 18 anos em cada ano em relação ao número total de alunos em cada escola.

Primeiramente, a pesquisa foi autorizada nos três municípios/países pelos órgãos competentes nas secretarias ou departamentos de educação. Para coleta de dados, utilizou-se um questionário, o qual foi avaliado e traduzido para o idioma espanhol. O questionário, elaborado especificamente para este estudo, passou por análise e validação de conteúdo e adaptação cultural por dois professores da área da educação em cada município / país.

Conduziu-se um estudo piloto, com sorteio de uma escola com 10 estudantes do equivalente ao ensino fundamental e médio de cada município/país para adequação do questionário com avaliação do conteúdo, estrutura e aplicabilidade em população com características semelhantes à população em estudo. Os participantes do estudo piloto não compuseram a amostra final.

O questionário contemplou 40 questões, subdivididas em informações sociodemográficas: sexo (masculino/ feminino), idade (anos), turno da aula (manhã, tarde, noite) e renda familiar em salários mínimos. Para o salário mínimo, foi considerado como referência o ano de 2012. Nesse ano, o salário mínimo no Brasil era de 622,00 reis (334,41 dólares); no Paraguai, era de 1658232 guaranis (435,11 dólares); e na Argentina, de 2670 pesos (575,40 dólares). Foram investigadas ainda as características da vida sexual: iniciação sexual (sim/não), idade da primeira relação sexual (anos), uso de método contraceptivo ( $\mathrm{sim} /$ não), recebimento de informações sobre métodos contraceptivos (sim/não), fonte de informação sobre os métodos contraceptivos (família, escola, jornal/revista, Internet, profissional de saúde, amigos/outros).

Para garantir a comparabilidade entre os países, realizou-se a uniformização entre os níveis escolares. Na equivalência dos níveis escolares, tomou-se o Brasil como referência para categorização dessa variável. Assim, o ensino fundamental $\left(7^{\circ}, 8^{\circ}\right.$ e $9^{\circ}$ anos) no Brasil correspondeu ao $3^{\mathrm{o}}$ ciclo no Paraguai $\left(7^{\mathrm{a}}, 8^{\mathrm{a}}\right.$ e $9^{\mathrm{a}}$ séries); na Argentina, correspondeu ao $7^{\circ}$ grau do ensino primário mais $1^{\mathrm{a}}$ e $2^{\mathrm{a}}$ séries do ensino secundário. Para o ensino médio, $1^{\circ}, 2^{\circ}$ e $3^{\circ}$ anos no Brasil corresponderam a $1^{\circ}, 2^{\circ}$ e $3^{\circ}$ anos de educação média secundária no Paraguai e a $3^{\mathrm{a}}, 4^{\mathrm{a}}$ e $5^{\mathrm{a}}$ séries do ensino secundário na Argentina.

A coleta de dados ocorreu nas instituições de ensino em horário de intervalo entre as aulas. O pesquisador responsável, acompanhado por professor e/ou pedagogo, procurou os estudantes, apresentou a proposta do estudo e os convidou a participar. $O$ questionário foi entregue para preenchimento pelos estudantes na presença do pesquisador no ambiente escolar. O período de coleta aconteceu nos anos de 2012 e 2013 nos três municípios.

O estudo foi aprovado pelo Comitê de Ética em Pesquisa da Universidade Estadual do Oeste do Paraná (UNIOESTE). Os aspectos éticos envolvendo pesquisa com seres humanos foram devidamente respeitados. Os pais ou responsáveis foram informados sobre a pesquisa por meio de uma carta entregue aos estudantes na escola. Posteriormente, os alunos cujos pais ou responsáveis consentiram com a participação trouxeram o termo de consentimento livre e esclarecido assinado pelos pais ou responsáveis. Para os alunos menores de 18 anos, houve também o consentimento por meio da assinatura do termo de assentimento.

As análises foram conduzidas utilizando-se o programa Statistical Package for the Social Sciences (SPSS) 17.0. 
Inicialmente, foram conduzidas análises descritivas de todas as variáveis, por meio de frequências absolutas e relativas. Posteriormente, foram conduzidas análises bivariadas dividida por município/país através dos testes do qui-quadrado $\left(\chi^{2}\right)$ de Pearson ou exato de Fisher para avaliar a associação entre já ter tido iniciação sexual e ter recebido informação sobre contraceptivos (variáveis dependentes) e as variáveis categóricas (independentes). Todas as análises foram realizadas em nível de significância de $5 \%$. Algumas questões não foram respondidas, levando a diferenças no número absoluto de respondentes apenas para Ciudad del Este/Paraguai, não gerando diferença estatística importante entre as variáveis analisadas.

\section{RESULTADOS}

Foram entrevistados 2788 adolescentes, sendo 1014 de Puerto Iguazú, 1071 de Foz do Iguaçu e 703 de Ciudad del Este. As perdas foram mínimas, por recusa em participar do estudo pelos pais ou responsáveis de adolescentes (figura 1).

Como mostra a tabela 1 , houve predomínio do sexo feminino no Brasil $(55,2 \%)$ e no Paraguai $(54,7 \%)$ e do sexo masculino na Argentina (48,8\%). Quanto ao perfil dos adolescentes, verificou-se que a maioria estava na faixa etária de 12 a 14 anos. A prevalência geral de iniciação sexual foi referida por $34,6 \%$ do total dos entrevistados. Entre os adolescentes argentinos, 45,3\% referiram já ter iniciado a vida sexual, sobressaindo-se em relação aos brasileiros (35,2\%) e aos paraguaios $(23,3 \%)$. Brasileiros e argentinos tiveram a primeira relação sexual mais precocemente do que paraguaios, na faixa etária de 12 a 14 anos (tabela 1). Quanto à iniciação sexual dos adolescentes em relação às características sociodemográficas, a análise bivariada mostrou as variáveis sexo, idade, escolaridade e turno de aula associadas à iniciação sexual nos três municípios, evidenciando a predominância do sexo masculino na iniciação sexual (tabela 2).

A maioria dos participantes referiu ter recebido informações sobre contraceptivos e fazer uso de contraceptivos no caso de relação sexual, com destaque para os adolescentes brasileiros (81,4\% e $81,6 \%$, respectivamente) (tabela 1 ). As meninas com idade de 12 a 14 anos foram as que mais haviam recebido informações sobre contraceptivos. A faixa etária de 17 a 18 anos foi a que menos recebeu essas informações. Nos três municípios, entre os que receberam informações sobre contraceptivos, $84,2 \%$ referiram o hábito de uso de contraceptivos $(P<0,001)$, sendo o preservativo o mais citado (Foz do Iguaçu: $64,8 \%$; Ciudad del Este: $68,8 \%$; Puerto Iguazú: 72,8\%). No município brasileiro, a combinação de preservativo e pílula anticoncepcional foi mais frequente $(22,0 \%)$. Nos outros municípios, o uso somente da pílula foi mais frequente (tabela 3).

A principal fonte de informação sobre métodos contraceptivos foi a família no Brasil e Argentina para o sexo feminino $(P<0,001)$. Já as paraguaias tiveram informações provenientes da família e escola $(P<0,001)$ (tabela 4$)$.

\section{DISCUSSÃO}

Este estudo mostrou que quase metade dos adolescentes dos municípios da tríplice fronteira entre Brasil, Argentina e Paraguai já haviam tido sua iniciação sexual, principalmente os do sexo masculino. Por outro lado, as adolescentes eram mais orientadas quanto aos métodos contraceptivos, especialmente pela família.

A contribuição deste trabalho consistiu em evidenciar a situação encontrada em região de tríplice fronteira pouco estudada, formada por municípios com

FIGURA 1. Fluxograma de seleção de participantes, estudo sobre iniciação sexual e práticas contraceptivas na tríplice fronteira Brasil, Argentina e Paraguaia

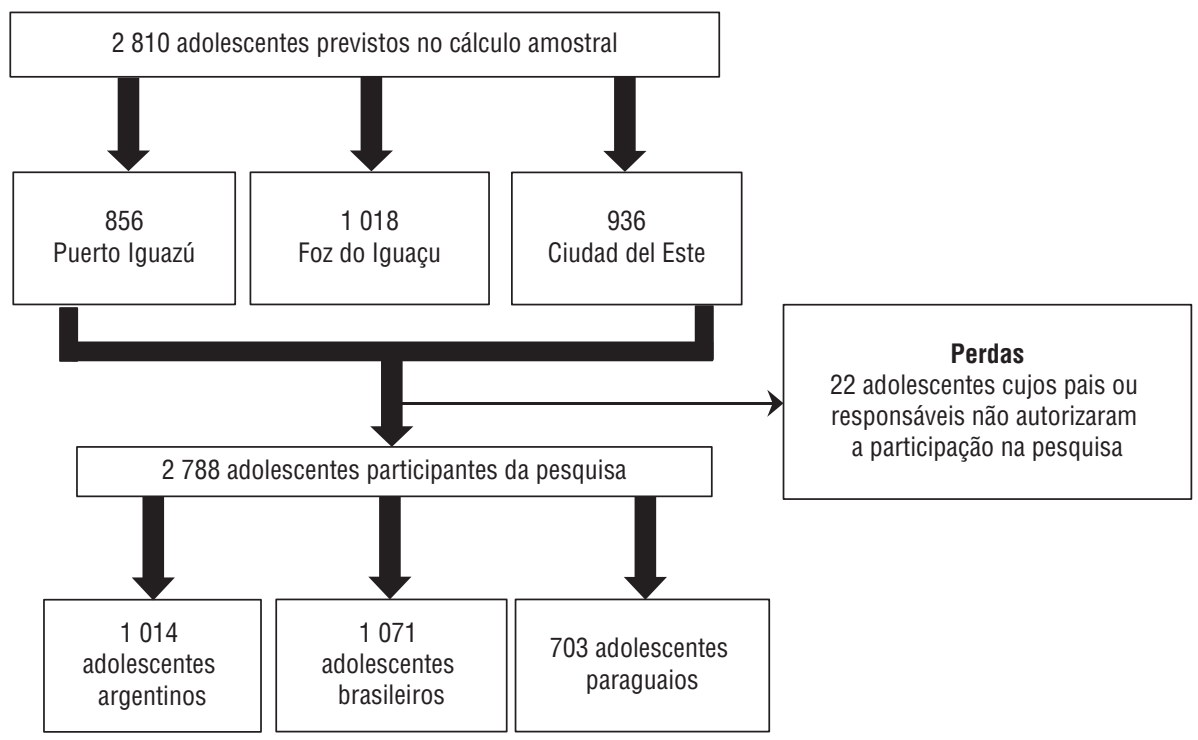

${ }^{a} \mathrm{Na}$ pesquisa, foram entrevistados $18,4 \%$ mais estudantes argentinos do que o previsto no cálculo amostral, assim como 5,2\% mais estudantes brasileiros do que o previsto no cálculo amostral. Assim, existe uma diferença entre o cálculo amostral e o número de participantes. realidades que se assemelham de certa forma por estarem situados em região com alta circulação de indivíduos. Considerou-se ainda que cada país tem uma política própria de saúde, com distintas formas de acesso ao sistema de saúde e cultura local (país), fatores esses que podem ter influenciados os resultados. Somada a isso, a constatação de poucos trabalhos na literatura internacional que retratem a temática na fronteira e a ausência desse tipo de estudo no Brasil também fortalecem este trabalho.

Comparações internacionais mostram que a prevalência da iniciação sexual varia entre países, sendo ligada a diferenças culturais importantes $(13,14)$. Quanto à distribuição por sexo, as meninas, neste estudo, tiveram iniciação sexual mais tardia do que os meninos, e em menor proporção. Foi observada associação estatística significativa para o sexo masculino, o que era esperado, visto se tratar de evento em que as relações de gênero atuam de forma inquestionável. Estudos mostram que, independentemente do lugar e do contexto sociocultural, os meninos tendem a apresentar maiores proporções de iniciação sexual em relação às meninas da mesma idade, com variações percentuais conforme a região $(15,16)$. Nesse sentido, a iniciação sexual parece não se tratar simplesmente de iniciativa individual e autônoma. Os rapazes, por questões sociais e culturais, sentem-se mais pressionados do 
TABELA 1. Características sociodemográficas e atividade sexual dos adolescentes em três cidades de fronteira, 2012 a 2013

\begin{tabular}{|c|c|c|c|c|c|c|}
\hline \multirow{2}{*}{ Variáveis sociodemográficas } & \multicolumn{2}{|c|}{ Foz do Iguaçu (Brasil) } & \multicolumn{2}{|c|}{ Ciudad del Este (Paraguai) } & \multicolumn{2}{|c|}{ Puerto Iguazú (Argentina) } \\
\hline & No. & $\%$ & No. & $\%$ & No. & $\%$ \\
\hline \multicolumn{7}{|l|}{ Sexo } \\
\hline Masculino & 447 & 44,8 & 317 & 45,3 & 489 & 51,2 \\
\hline \multicolumn{7}{|l|}{ Idade (anos) } \\
\hline 12 a 14 & 493 & 47,9 & 360 & 50,5 & 488 & 48,4 \\
\hline \multicolumn{7}{|l|}{ Escolaridade } \\
\hline Ensino fundamental Brasil & 574 & 56,2 & & & & \\
\hline Ensino médio Brasil & 449 & 43,8 & & & & \\
\hline Educação básica Paraguai & & & 439 & 63,0 & & \\
\hline Educação média Paraguai & & & 257 & 37,0 & & \\
\hline Ensino primário Argentina & & & & & 709 & 80,5 \\
\hline Tarde & 288 & 29,1 & 334 & 48,1 & 301 & 32,8 \\
\hline Noite & 214 & 21,6 & 86 & 12,4 & 128 & 13,9 \\
\hline \multicolumn{7}{|l|}{ Renda familiar (salários mínimos) ${ }^{\mathrm{a}}$} \\
\hline$<1$ & 178 & 18,1 & 280 & 44,9 & 116 & 48,1 \\
\hline 1 a 3 & 612 & 62,6 & 278 & 44,6 & 97 & 40,2 \\
\hline 4 a 5 & 145 & 14,8 & 37 & 5,9 & 18 & 7,5 \\
\hline$>5$ & 44 & 4,5 & 28 & 4,6 & 10 & 4,2 \\
\hline \multicolumn{7}{|l|}{ Características da atividade sexual } \\
\hline \multicolumn{7}{|l|}{ Iniciou a vida sexual } \\
\hline Sim & 358 & 35,2 & 164 & 23,3 & 443 & 45,3 \\
\hline Não & 658 & 64,8 & 539 & 76,7 & 535 & 54,7 \\
\hline Não & 63 & 18,4 & 34 & 20,7 & 84 & 21,2 \\
\hline \multicolumn{7}{|l|}{ Método contraceptivo em relação sexual } \\
\hline Preservativo & 167 & 66,5 & 82 & 70,1 & 204 & 74,7 \\
\hline Preservativo e pílula anticoncepcional & 50 & 19,9 & 10 & 8,5 & 21 & 7,7 \\
\hline Pílula anticoncepcional & 31 & 12,4 & 14 & 12,0 & 35 & 12,8 \\
\hline Outros métodos & 03 & 1,2 & 11 & 9,4 & 13 & 4,8 \\
\hline \multicolumn{7}{|l|}{ Recebeu informação sobre contraceptivos } \\
\hline Sim & 821 & 81,4 & 502 & 72,3 & 684 & 70,8 \\
\hline Não & 187 & 18,6 & 192 & 27,7 & 282 & 29,2 \\
\hline
\end{tabular}

a Salário mínimo: 2 670,00 pesos/575,40 dólares na Argentina; 622,00 reais/334,41 dólares no Brasil; 1658 232,00 guaranis/435,11 dólares no Paraguai. As categorias apresentadas levam em conta o salário mínimo de cada país.

que as moças a deixarem de ser virgens, sendo maior a pressão externa atribuída aos amigos. O relato das adolescentes pode refletir a ambivalência entre o desejo e a norma social ainda vigente em alguns contextos sociais, que considera a iniciativa como prerrogativa masculina, devendo as mulheres resistir para posteriormente ceder (14). A investigação de estratégias de acomodação e resistência aos estereótipos dos papéis sexuais desempenhados é fundamental, considerando as questões de gênero (16).

Em estudo nacional no Brasil, foi apontado que mais de um quinto dos adolescentes de 12 a 17 anos de idade já haviam iniciado a vida sexual. Quanto a isso, a prevalência de iniciação sexual foi bem mais baixa nos indivíduos com 12 a 14 anos em relação aos com idade superior, o que revela tendência ao aumento da prevalência de iniciação sexual conforme aumenta a idade (17). Um relatório do Fundo das Nações Unidas para a Infância (UNICEF) (18) na Argentina apontou a idade média de iniciação sexual dos meninos como 15 anos, próximo ao 
TABELA 2. Análise bivariada da associação entre ter tido iniciação sexual e variáveis sociodemográficas em adolescentes de três cidades de fronteira, 2012 a 2013

\begin{tabular}{|c|c|c|c|c|c|c|c|c|c|}
\hline \multirow{4}{*}{ Variáveis Sociodemográficas } & \multicolumn{9}{|c|}{ Iniciação sexual } \\
\hline & \multicolumn{3}{|c|}{ Foz do Iguaçu (Brasil) } & \multicolumn{3}{|c|}{ Ciudad del Este (Paraguai) } & \multicolumn{3}{|c|}{ Puerto Iguazú (Argentina) } \\
\hline & \multicolumn{3}{|c|}{ Sim } & \multicolumn{3}{|c|}{ Sim } & \multicolumn{3}{|c|}{$\operatorname{Sim}$} \\
\hline & No. & $\%$ & $P$ & No. & $\%$ & $P$ & No. & $\%$ & $P$ \\
\hline \multicolumn{10}{|l|}{ Sexo } \\
\hline Masculino & 190 & 55,4 & $<0,001$ & 92 & 57,5 & $<0,001$ & 254 & 60,7 & $<0,001$ \\
\hline Feminino & 153 & 44,6 & & 68 & 42,5 & & 164 & 39,3 & \\
\hline 15 a 16 & 150 & 41,9 & & 69 & 43,1 & & 192 & 43,5 & \\
\hline 17 a 18 & 135 & 37,7 & & 72 & 45,0 & & 135 & 30,6 & \\
\hline \multicolumn{10}{|l|}{ Escolaridade } \\
\hline Ensino fundamental Brasil & 128 & 36,0 & $<0,001$ & & & & & & \\
\hline Ensino médio Brasil & 227 & 64,0 & & & & & & & \\
\hline \multicolumn{10}{|l|}{ Período da aula } \\
\hline Manhã & 141 & 40,9 & $<0,001$ & 55 & 34,8 & $<0,001$ & 171 & 42,6 & $<0,001$ \\
\hline Tarde & 63 & 18,4 & & 60 & 38,0 & & 136 & 33,9 & \\
\hline Noite & 140 & 40,7 & & 43 & 27,2 & & 94 & 23,5 & \\
\hline \multicolumn{10}{|l|}{ Renda familiara (salários mínimos) } \\
\hline$<1$ & 60 & 17,1 & 0,048 & 68 & 46,6 & 0,369 & 71 & 51,5 & 0,501 \\
\hline 1 a 3 & 213 & 60,8 & & 61 & 41,8 & & 51 & 36,9 & \\
\hline 4 a 5 & 53 & 15,2 & & 7 & 4,8 & & 11 & 7,9 & \\
\hline$>5$ & 24 & 6,9 & & 10 & 6,8 & & 5 & 3,7 & \\
\hline
\end{tabular}

a Salário mínimo: 2 670,00 pesos/575,40 dólares na Argentina; 622,00 reais/334,41 dólares no Brasil; 1658 232,00 guaranis/435,11 dólares no Paraguai. As categorias apresentadas levam em conta o salário mínimo de cada país.

referido pelos participantes deste estudo. No mesmo país, uma investigação com adolescentes e jovens do sexo feminino mostrou que a iniciação sexual ocorreu predominantemente entre 15 e 17 anos, e uma minoria antes dos 14 anos (16). Uma pesquisa com militares paraguaios de ambos os sexos apontou média da iniciação sexual aos 16 anos (19), o que difere dos estudantes paraguaios neste estudo, que referiram idade entre 17 e 18 anos. Essas diferenças etárias quanto ao sexo poderiam estar relacionadas ao padrão social de repressão da sexualidade feminina, o que poderia levar ao sub-relato da idade de iniciação sexual por elas. Para o sexo masculino, espera-se a atividade sexual e a satisfação de desejos sexuais como requisitos de masculinidade, o que contribui para o relato ou mesmo o exagero nesse relato para idades mais precoces (15).

A prevalência da iniciação sexual entre os adolescentes mais jovens (12 a 14 anos) é preocupante e deve ser alvo também de investigações. $O$ início de atividade sexual anterior aos 14 anos geralmente está ligado à paternidade na adolescência, o que pode ter importante impacto na vida dos adolescentes (20). Esse público deve ser alvo da formulação de políticas, programas e práticas de saúde que envolvam direitos sexuais e reprodutivos para subsidiar o início da vida sexual saudável, responsável e livre de qualquer coerção (15).

Os resultados deste estudo evidenciaram relação direta entre maior escolaridade do adolescente e ter tido iniciação sexual em Foz do Iguaçu e Ciudad del Este, o que difere de outro trabalho com adolescentes mais velhos (16). Acreditase que a escolaridade mais alta dos entrevistados possa refletir um maior tempo sob influência de pressões de amigos e colegas e de normas, escolhas e expectativas sociais vivenciadas por esses indivíduos em fases anteriores da adolescência, o que pode justificar a maior iniciação sexual desse público.

Outro ponto importante no estudo foi a relação inversa entre escolaridade do adolescente e o recebimento de informações sobre contraceptivos nos três municípios. Os adolescentes de menor escolaridade receberam mais informações sobre a temática, com destaque para os estudantes de Puerto Iguazú. O recebimento de tais informações foi menor entre o sexo masculino, que também foi o grupo que referiu maior percentual de iniciação sexual e de maneira mais precoce, sendo menos adepto ao uso de contraceptivos. Salienta-se que a educação sexual e o acesso à informação devem ser primordiais nesta etapa da vida, como apontam outras investigações (19-21).

Similarmente, uma investigação realizada no Paraguai mostrou que adolescentes do sexo masculino referiram uso de preservativo em menor proporção e, mais que isso, quanto maior a idade, menor era a propensão ao uso de preservativo (19). No que se refere ao menor acesso a informações entre indivíduos e a propensão ao não uso de contraceptivos pelo sexo masculino, aponta-se para a necessidade de abordagem sobre conhecimentos, 
TABELA 3. Análise bivariada da associação entre ter recebido informação sobre método contraceptivo e variáveis sociodemográficas/uso de contraceptivo em adolescentes de três cidades de fronteira, 2012 a 2013

\begin{tabular}{|c|c|c|c|c|c|c|c|c|c|}
\hline \multirow{4}{*}{ Variável } & \multicolumn{9}{|c|}{ Informado sobre contraceptivos } \\
\hline & \multicolumn{3}{|c|}{ Foz do Iguaçu (Brasil) } & \multicolumn{3}{|c|}{ Ciudad del Este (Paraguai) } & \multicolumn{3}{|c|}{ Puerto Iguazú (Argentina) } \\
\hline & \multicolumn{3}{|c|}{$\operatorname{Sim}$} & \multicolumn{3}{|c|}{$\operatorname{Sim}$} & \multicolumn{3}{|c|}{$\operatorname{Sim}$} \\
\hline & No. & $\%$ & $P$ & No. & $\%$ & $P$ & No. & $\%$ & $P$ \\
\hline \multicolumn{10}{|l|}{ Sexo } \\
\hline Masculino & 329 & 41,2 & $<0,001$ & 205 & 41,9 & 0,004 & 294 & 45,6 & $<0,001$ \\
\hline Feminino & 468 & 58,8 & & 284 & 58,1 & & 350 & 54,4 & \\
\hline \multicolumn{10}{|l|}{ Idade (anos) } \\
\hline 12 a 14 & 356 & 43,3 & $<0,001$ & 207 & 41,9 & $<0,001$ & 280 & 41,2 & $<0,001$ \\
\hline 15 a 16 & 305 & 37,1 & & 173 & 35 & & 256 & 37,7 & \\
\hline 17 a 18 & 161 & 19,6 & & 114 & 23,1 & & 143 & 21,1 & \\
\hline \multicolumn{10}{|l|}{ Escolaridade } \\
\hline Ensino fundamental Brasil & 412 & 50,6 & $<0,001$ & & & & & & \\
\hline Ensino médio Brasil & 402 & 49,4 & & & & & & & \\
\hline Ensino fundamental Paraguai & & & & 271 & 55,8 & $<0,001$ & & & \\
\hline Ensino médio Paraguai & & & & 214 & 44,2 & & & & \\
\hline Ensino primário Argentina & & & & & & & 454 & 76,0 & $<0,001$ \\
\hline Ensino secundário Argentina & & & & & & & 143 & 24,0 & \\
\hline \multicolumn{10}{|l|}{ Período da aula } \\
\hline Manhã & 384 & 48,7 & $<0,001$ & 180 & 37,5 & 0,033 & 315 & 50,6 & 0,075 \\
\hline Tarde & 214 & 27,1 & & 233 & 48,5 & & 217 & 34,8 & \\
\hline Noite & 191 & 24,2 & & 67 & 14,0 & & 91 & 14,6 & \\
\hline \multicolumn{10}{|l|}{ Renda (salários mínimos)a } \\
\hline$<1$ & 128 & 16,3 & 0,058 & 187 & 42,6 & 0,493 & 86 & 47,5 & 0,691 \\
\hline 1 a 3 & 498 & 63,6 & & 203 & 46,3 & & 72 & 39,8 & \\
\hline 4 a 5 & 120 & 15,3 & & 26 & 5,9 & & 16 & 8,8 & \\
\hline$>5$ & 38 & 4,8 & & 23 & 5,2 & & 7 & 3,9 & \\
\hline \multicolumn{10}{|l|}{ Uso de contraceptivo } \\
\hline $\operatorname{Sim}$ & 254 & 84,3 & $<0,001$ & 121 & 84,0 & $<0,001$ & 255 & 84,1 & $<0,001$ \\
\hline Não & 47 & 15,7 & & 23 & 16,0 & & 48 & 15,9 & \\
\hline \multicolumn{10}{|l|}{ Método contraceptivo } \\
\hline Preservativo & 149 & 64,8 & 0,108 & 75 & 68,8 & 0,528 & 166 & 72,9 & 0,695 \\
\hline Preservativo e pílula anticoncepcional & 51 & 22,1 & & 9 & 8,3 & & 18 & 7,8 & \\
\hline Pílula anticoncepcional & 27 & 11,7 & & 14 & 12,8 & & 32 & 14,1 & \\
\hline Outros métodos & 3 & 1,3 & & 11 & 10,1 & & 12 & 5,2 & \\
\hline
\end{tabular}

a Salário mínimo: 2 670,00 pesos/575,40 dólares na Argentina; 622,00 reais/334,41 dólares no Brasil; 1658 232,00 guaranis/435,11 dólares no Paraguai. As categorias apresentadas levam em conta o salário mínimo de cada país.

atitudes e crenças para este público, a fim de que ocorra prevenção de consequências reprodutivas não intencionais (22).

É preocupante que a faixa etária que menos referiu ter recebido orientações sobre contraceptivos (17 a 18 anos) neste estudo inclui-se entre os que tiveram maior proporção de iniciação sexual nos três municípios/países (16 a 18 anos). Nesse sentido, parecem existir lacunas no processo formativo e educativo dos adolescentes nas referidas cidades, o que pode ter como resultado o baixo empoderamento dos indivíduos para tomada de decisões acerca da saúde sexual e reprodutiva. Uma revisão sistemática sobre essa temática, envolvendo países de baixa e média renda, apontou a falta de conhecimento dos adolescentes sobre infecções sexualmente transmissíveis e também a procura reduzida por serviços especializados por essa faixa etária. Nesse caso, os motivos para a baixa procura por serviços de saúde foram relacionados a barreiras, como vergonha, incerteza sobre a confidencialidade ou sensação de pouco acolhimento pelos profissionais de saúde (23).

Uma investigação com brasileiros em Montes Claros, no estado de Minas Gerais (região Sudeste), verificou que os adolescentes com mais de 16 anos possuíam conhecimento mais adequado sobre contraceptivos e faziam o uso dos mesmos (24). Em contrapartida, outros estudos conduzidos no Maranhão, Nordeste do Brasil, destacam que vários adolescentes mencionaram desconhecer ou ter conhecimento insuficiente da maioria dos métodos contraceptivos (25), ressaltando a necessidade de maior enfoque no ensino por profissionais de saúde, escola e família $(26,27)$.

Os resultados do presente estudo demonstraram que as fontes de informação dos adolescentes foram principalmente a família e a escola, levantando um questionamento sobre a atuação dos profissionais de saúde, que pode ter sido restrita. Este resultado é comparável aos de outros trabalhos desenvolvidos no Brasil, nos quais os amigos foram a fonte de informação mais relatada (28), sendo 
TABELA 4. Análise bivariada da associação entre fonte de informação sobre métodos contraceptivos e realização de sexo antes dos 18 anos em adolescentes de três cidades de fronteira, 2012 a 2013

\begin{tabular}{|c|c|c|c|c|c|c|c|c|c|c|c|c|c|c|c|}
\hline \multirow{3}{*}{ Fonte de informação ${ }^{a}$} & \multicolumn{5}{|c|}{ Foz do Iguaçu (Brasil) } & \multicolumn{5}{|c|}{ Ciudad del Este (Paraguai) } & \multicolumn{5}{|c|}{ Puerto Iguazú (Argentina) } \\
\hline & \multicolumn{2}{|c|}{ Masculino } & \multicolumn{2}{|c|}{ Feminino } & \multirow[b]{2}{*}{$P$} & \multicolumn{2}{|c|}{ Masculino } & \multicolumn{2}{|c|}{ Feminino } & \multirow[b]{2}{*}{$P$} & \multicolumn{5}{|c|}{ Feminino } \\
\hline & No. & $\%$ & No. & $\%$ & & No. & $\%$ & No. & $\%$ & & No. & $\%$ & No. & $\%$ & $P$ \\
\hline Sim & 163 & 36,3 & 293 & 53,1 & $<0,001$ & 109 & 34,3 & 175 & 45,6 & 0,002 & 164 & 33,5 & 247 & 52,8 & $<0,001$ \\
\hline \multicolumn{16}{|l|}{ Escola } \\
\hline Sim & 148 & 33,1 & 199 & 36,1 & 0,178 & 85 & 26,8 & 127 & 33,1 & 0,041 & 128 & 26,1 & 168 & 35,9 & 0,001 \\
\hline \multicolumn{16}{|l|}{ Jornal } \\
\hline Sim & 25 & 5,6 & 36 & 6,5 & 0,315 & 10 & 3,1 & 16 & 4,2 & 0,306 & 17 & 3,4 & 35 & 7,5 & 0,005 \\
\hline \multicolumn{16}{|l|}{ Internet } \\
\hline Sim & 34 & 7,6 & 39 & 7,0 & 0,421 & 18 & 5,6 & 11 & 2,8 & 0,048 & 38 & 7,7 & 46 & 9.8 & 0,154 \\
\hline \multicolumn{16}{|l|}{ Profissional de saúde } \\
\hline
\end{tabular}

o profissional de saúde pouco referido como fonte de informação (25).

Um estudo em Goiânia (24), no Centro-Oeste brasileiro, mostrou a necessidade de parcerias entre as áreas da saúde e da educação, em especial pela atuação de enfermeiros no ensino e orientação à saúde sexual e reprodutiva, tendo suporte didático-pedagógico de professores da rede básica de ensino para o trabalho de orientação sobre a sexualidade envolvendo alunos adolescentes.

Na rede municipal de ensino da cidade de São Paulo, notou-se a concepção descontextualizada da sexualidade e da promoção de saúde. Nessa cidade, não se observou trabalho processual, interdisciplinar e intersetorial. $\mathrm{O}$ encontrado foram ações pontuais e informativas em aulas de ciências e em palestras esporádicas com profissionais de saúde (22).

A orientação adequada sobre iniciação sexual, relacionamentos e prevenção de infeções sexualmente transmissíveis impede os comportamentos sexuais de risco e estimula a prática do sexo responsável, aumentando a sensibilização e permitindo o questionamento de regras. Isso ajuda na compreensão das informações adquiridas e pode influenciar a tomada de decisões (13).

$\mathrm{O}$ acesso a programas intersetoriais, especialmente aqueles voltados à educação e à saúde, é fundamental para que a população adolescente vivencie a sua sexualidade de forma mais plena e saudável e para mudança nas dinâmicas de gênero em toda a sociedade. No Brasil, o Programa Saúde na Escola é uma estratégia que propicia a integração entre saúde e educação e que pode propiciar a educação sexual dos adolescentes. Orienta-se que o ensino sobre saúde sexual e sexualidade seja trabalhado desde o primeiro ciclo da educação formal com educadores, organizações não governamentais, conselhos e, sobretudo, com profissionais que atuam na área da saúde (25).

O presente estudo tem algumas limitações. Por possuir delineamento transversal, torna-se limitado para estabelecer causa e efeito, além de estar sujeito à possibilidade de viés de memória. Também não inclui uma análise da qualidade das informações repassadas, ou que os adolescentes tinham sobre contraceptivos. Participaram do estudo somente adolescentes de escolas públicas, o que não permite extrapolar os resultados para todos os estudantes adolescentes da tríplice fronteira. Trata-se de investigação realizada na tríplice fronteira, a qual apresenta suas particularidades em relação às outras cidades da região. Ainda, considerando que não existem estudos que abordem os três países na perspectiva comparada sobre esse tema na fronteira, é fundamental que novas pesquisas sejam desenvolvidas abordando a orientação sobre métodos contraceptivos e iniciação sexual nestas nações.
Por fim, a amostra populacional, composta por adolescentes estudantes de escolas públicas, permitiu comparar aspectos entre práticas contraceptivas e iniciação sexual na tríplice fronteira. É de fundamental importância que os profissionais de saúde se insiram num trabalho interdisciplinar com o intuito de planejar estratégias que permitam melhorias no serviço de saúde e, consequentemente, impliquem na promoção da qualidade de vida dos adolescentes. Os achados deste estudo sugerem a necessidade de políticas de fronteira que possam garantir direitos humanos básicos, com aprimoramento de programas e projetos voltados à orientação sobre saúde sexual e reprodutiva que contemplem o atendimento de população fronteiriça de adolescentes.

Agradecimentos. O estudo foi financiado por Itaipu binacional (termo de compromisso número 4500011787) e bolsa de estudo (EMTPP) da Fundação Araucária (convênio número 305/2010).

Conflitos de interesse. Nada declarado pelos autores.

Declaração. As opiniões expressas no manuscrito são de responsabilidade exclusiva dos autores e não refletem necessariamente a opinião ou política da RPSP/ PAJPH ou da Organização Pan-americana de Saúde (OPAS). 


\section{REFERÊNCIAS}

1. Patton GC, Sawyer SM, Santelli JS, Ross DA, Afifi R, Allen NB, et al. Our future: a Lancet commission on adolescent health and wellbeing. Lancet. 2016;387(10036): 2423-78.

2. Santhya KG, Jejeebhoy SJ. Sexual and reproductive health and rights of adolescent girls: evidence from low- and middle-income countries. Glob Public Health. 2015;10(2):189-221.

3. Marinho LF, Aquino EM, Almeida MCC. Práticas contraceptivas e iniciação sexual entre jovens de três capitais brasileiras. Cad Saude Publica. 2009;25 Suppl 2:227-39.

4. World Health Organization (WHO). The sexual and reproductive health of young adolescents in developing countries: reviewing the evidence, identifying research gaps, and moving the agenda. Report of a WHO technical consultation. Genebra, 4-5 de novembro de 2010. Disponível em: http:/ / apps.who.int/iris/bitstream / 10665/70569/1/WHO_RHR_11.11_eng. pdf Acessado em 8 de outubro de 2016.

5. Taquette SR. Doenças sexualmente transmissíveis em adolescentes femininas de comunidades pobres do município do Rio de Janeiro: incidência e diferenças de raça/cor na vulnerabilidade às DST/Aids. Adolesc Saude. 2011;8(3):18-26.

6. Kann L, Kinchen S, Shanklin SL, Flint KH, Kawkins J, Harris WA, et al. Youth Risk Behavior Surveillance - United States, 2013. MMWR. 2014;63(4):1-168.

7. Vignoli JR. Adolescent fertility in Latin America and the Caribbean. 2016. Disponível em: http://www.niussp. org/2016/05/09/adolescent-fertility-in-latin-america-and-the-caribbeanla-fecondite-des-adolescentes-en-amerique-latine-et-dans-les-antilles /?print=pdf Acessado em 20 de outubro de 2016.

8. Joint United Nations Program on HIV/ AIDS. Prevention gap report. Genebra: UNAIDS; 2016. Disponível em: http:// www.unaids.org/sites/default/files/media_asset/2016-prevention-gap-report_ en.pdf Acessado em 20 de outubro de 2016.

9. Lima FRF. Reflexões sobre o processo de ocupação da tríplice fronteira - Brasil, Argentina e Paraguai. Rev Geogr (UFPE). 2015;32(3):32-42.

10. United Nations Children's Fund. Convención sobre los derechos del niño.
Madri: UNICEF Comité Español; 2016. Disponível em: http://www.un.org/es/ events / childrenday/pdf/derechos.pdf Acessado em 20 de dezembro de 2016.

11. Brasil. Lei 8 069/1990. Disponível em: http:/ / www.planalto.gov.br/ccivil_03/ leis/L8069.htm Acessado em 17 de agosto de 2017.

12. Red Latinoamericana de Acogimiento Familiar (RELAF). Legislaciones de niñez. Latinoamericana. 2015. Disponível em: http://www.relaf.org/legislaciones2.htm Acessado em 16 de dezembro de 2017.

13. Oliveira-Campos M, Nunes ML, Madeira FC, Santos MG, Bregmann SR, Malta DC, et al. Comportamento sexual em adolescentes brasileiros, Pesquisa Nacional de Saúde do Escolar (PeNSE 2012). Rev Bras Epidemiol. SupplPeNSE. 2014;116-30.

14. Gonçalves H, Machado EC, Soares ALG, Camargo-Figueira FA, Seering LM, Mesenburg MA, et al. Início da vida sexual entre adolescentes (10 a 14 anos) e comportamentos em saúde. Rev Bras Epidemiol. 2015;18(1):25-41.

15. Marinho LFB, Aquino EML, Almeida MCC. Práticas contraceptivas e iniciação sexual entre jovens de três capitais brasileiras. Cad Saude Publica. 2009;25(Suppl 2):227-39.

16. Abdul Samad S, Hairi NN, Ismail M. Role of individual, family, and peers in sexual initiation among late adolescents attending institutions of higher learning in Malaysia. Asia Pac J Public Health. 2016;28(4):313-24.

17. Borges ALV, Fujimori E, Kuschnir MCC, Chofakian CBN, Moraes AJP, Azevedo GD, et al. ERICA: início da vida sexual e contracepção em adolescentes brasileiros. Rev Saude Publica. 2016;50(supl 1):15s.

18. Fundación Huésped, UNICEF Argentina. Conocimientos, actitudes y prácticas en VIH y salud sexual y reproductiva (SSR) y uso de tecnologías de la información entre adolescentes de Argentina. Buenos Aires: UNICEF; 2012.

19. Laguna-Torres VA, Aquayo N, Aguilar G, Ampuero JS, Galeano A, Barboza A, et al. HIV infection and high-risk behaviours in a Paraguayan military population. Int J STD AIDS. 2014;25(12):866-77.

20. Silva MAI, Mello FCM, Mello DF, Ferriani MGC, Sampaio JMC, Oliveira WA Vulnerabilidade na saúde do adolescente: questões contemporâneas. Cienc Saude Coletiva. 2014;19(2):619-27.

21. Binstock G, Gogna M. La iniciación sexual entre mujeres de sectores vulnerables en cuatro provincias argentinas. Sex Salud Soc (Rio J). 2015;20:113-40.

22. Pirotta KCM, Barboza R, Pupo LR, Unbehaum S, Cavasin S. Programas de orientação sexual nas escolas: uma análise das lacunas na implementação de políticas públicas a partir da percepção dos alunos da rede municipal de ensino de São Paulo. Rev Gest Polit Publicas. 2013;3(1): 190-210.

23. Carneiro RF, Silva NC, Alves TA, Albuquerque DO, Brito DC, Oliveira LL. Educação sexual na adolescência: uma abordagem no contexto escolar. SANARE. 2015;14(1):104-8.

24. Rufino CB, Pires LM, Oliveira PC, Souza SMB, Souza MM. Educação sexual na prática pedagógica de professores da rede básica de ensino. Rev Eletr Enf. 2013;15(4):983-91.

25. Vieira EL, Pessoa GRS, Vieira LL, Carvalho WRC, Firmo WCA. Uso e conhecimento sobre métodos contraceptivos de estudantes da rede de ensino pública e privada do município de Bacabal-MA. Rev Cientifica ITPAC. 2016:9(2):88-106.

26. Carvalho MTVF, Batista APL, Figueiredo MFS, Barbosa AAD, Marinho LM, Caitite LC. Conhecimento dos adolescentes de escolas públicas de Montes Claros acerca do uso de métodos contraceptivos. Rev Norte Min Enferm. 2012;1(1):33-44.

27. Abtibol CS, Rocha FCG, Silva MGP, Silva VA, Oliveira FDS, Carvalho ML. Conhecimento de adolescentes de uma escola pública sobre os métodos contraceptivos. Rev Interd. 2015;8(2):94-100.

28. Araújo MSP, Costa LOBF. Comportamento sexual e contracepção de emergência entre adolescentes de escolas públicas de Pernambuco, Brasil. Cad Saude Publica. 2009;25(3):551-62.

Manuscrito recebido em 25 de maio de 2017. Aceito em versão revisada em 29 de agosto de 2017 
ABSTRACT Objective. To determine the prevalence of sexual initiation and the contraceptive practices of adolescents in the tri-border area between the cities of Foz do Iguaçu (Brazil), Ciudad del Este (Paraguay), and Puerto Iguazú (Argentina).

Sexual initiation and contraceptive practices among adolescents in the tri-border area between Brazil, Argentina, and Paraguay

Keywords Sexual behavior; border areas; contraception behavior; Argentina; Brazil; Paraguay.
Method. A cross-sectional study was conducted with 2788 adolescents from public schools (age 12 to 18 years). Information was obtained through questionnaires administered in the schools between 2012 and 2013. The prevalence of sexual initiation, contraceptive practices, and sociodemographic variables was analyzed. To verify the association between the studied variables, the chi-square test was used with a significance level of $5 \%$.

Results. The overall prevalence of sexual initiation among adolescents was $34.6 \%$ (Puerto Iguazú: 45.3\%; Foz do Iguaçu: 35.2\%; Ciudad del Este: 23.3\%). The prevalence of sexual initiation was higher in males and in the 15-16 year age range. Sexual initiation was associated with sex, age, schooling, and school shift (morning, afternoon, or evening). Having received information on contraceptives was associated with sex, age, schooling, and contraceptive use in all three cities/countries. Adolescents of both sexes had received information about contraceptives, especially from the family and the school.

Conclusions. It is suggested that municipalities/countries seek to structure a tri-national border policy with intersectoral programs and projects in order to promote educational practices regarding sexual and reproductive health for adolescents.
RESUMEN

\section{Iniciación sexual y prácticas anticonceptivas de adolescentes en la triple frontera entre Brasil, Argentina y Paraguay}

Palabras clave
Objetivo. Determinar la prevalencia de adolescentes iniciados sexualmente y sus prácticas anticonceptivas en la región de la triple frontera entre Foz do Iguaçu (Brasil), Ciudad del Este (Paraguay) y Puerto Iguazú (Argentina).

Método. Estudio transversal en el cual participaron 2788 adolescentes de escuelas públicas, con edades comprendidas entre 12 y 18 años. La información fue obtenida a través de cuestionarios aplicados en las escuelas durante el período de 2012-2013. Se analizaron la prevalencia de iniciación sexual, prácticas anticonceptivas y variables sociodemográficas de individuos de los tres municipios. Para verificar la asociación entre las variables, se utilizó el chi-cuadrado con un nivel de significación del 5\%.

Resultados. La prevalencia general de iniciación sexual de los adolescentes fue del 34,6\% (Puerto Iguazú: 45,3\%, Foz do Iguaçu: 35,2\%, Ciudad del Este: 23,3\%), siendo mayor en el sexo masculino y en la franja etaria de 15 a 16 años. En cuanto a ya haber tenido iniciación sexual, ese factor se asoció al sexo, la edad, la escolaridad y el turno de clase de los participantes. La recepción de información sobre anticonceptivos se asoció con el sexo, la edad, la escolaridad y el uso de anticonceptivos en los tres municipios/países. Se observó que los adolescentes de ambos sexos habían recibido información sobre anticonceptivos, principalmente a partir de la familia y de la escuela. Conclusiones. Se sugiere que los municipios busquen la estructuración de una política de frontera trinacional con programas y proyectos intersectoriales con el fin de promover prácticas educativas dirigidas a la orientación de la salud sexual y reproductiva de los adolescentes.

Conducta sexual; áreas fronterizas; conducta anticonceptiva; Argentina; Brasil; Paraguay. 